\title{
Tunneling-induced giant Goos-Hänchen shift in quantum wells
}

\author{
Wen-Xing Yang, ${ }^{1,2, *}$ Shaopeng Liu, ${ }^{1}$ Zhonghu Zhu, ${ }^{1}$ Ziauddin, $^{2,3}$ and Ray-Kuang Lee ${ }^{2}$ \\ ${ }^{1}$ Department of Physics, Southeast University, Nanjing 211189, China \\ ${ }^{2}$ Institute of Photonics Technologies, National Tsing-Hua University, Hsinchu 300, Taiwan \\ ${ }^{3}$ Department of Physics, COMSATS Institute of Information Technology, Islamabad 44000, Pakistan \\ *Corresponding author: wenxingyang@seu.edu.cn
}

Received 8 May 2015; revised 28 May 2015; accepted 1 June 2015; posted 9 June 2015 (Doc. ID 240540 ); published 26 June 2015

Tunneling-induced quantum interference experienced by an incident probe in the asymmetric double AlGaAs/GaAs quantum well $(\mathrm{QW})$ structure can be modulated by means of an external control light beam and the tunable coupling strengths of resonant tunneling. These phenomena can be exploited to devise a novel intracavity medium to control Goos-Hänchen $(\mathrm{GH})$ shifts of a mid-infrared probe beam incident on a cavity. For a suitably designed QW structure, our results show that maximum negative shift of $2.62 \mathrm{~mm}$ and positive shift of $0.56 \mathrm{~mm}$ are achievable for GH shifts in the reflected and transmitted light. @ 2015 Optical Society of America

OCIS codes: (190.5970) Semiconductor nonlinear optics including MQW; (270.1670) Coherent optical effects; (260.3160) Interference.

http://dx.doi.org/10.1364/OL.40.003133

The Goos-Hänchen (GH) effect happens when a linearly polarized light undergoes a small lateral shift for the totally internal reflection from the interface of two different media [1]. The observation by Goos and Hänchen in 1947 has attracted considerable interest because of a variety of applications in optical heterodyne sensing and precision measurement, such as refractive index, displacement, temperature, and film thickness [2-4]. Interesting proposals toward the control or enhancement of $\overline{\mathrm{G}} \mathrm{H}$ shift have already been proposed to have negative and positive GH shifts [5-9]].

In this Letter, we present a proof-of-principle investigation by demonstrating that tunneling-induced quantum interference in a semiconductor quantum well (QW) structure can be exploited to provide an efficient new mechanism for enhancing and controlling GH shift in the reflected and transmitted light. Compared to the scenario when the control beam is off, the GH shift can be reduced significantly in the presence of an external control beam. The different responses depend on whether the tunneling-induced interference is quenched or well developed. Quantum interference-based phenomena, such as electromagnetically induced transparency (EIT) $[\underline{10}, 11]$, lasing without population inversion [12,13], and Raman gain process
[14,15] have attracted considerable attention. Simultaneously, these phenomena also are extended to a variety of applications $[15,16]$. Different from the mechanism of controlling GH shift based on photon-induced changes of the medium refractive index via coherent control beam [5-9], the mechanism of control $\mathrm{GH}$ shift is achieved here as a result of the tunneling-induced quantum interference [17-23].

The schematic of a weak probe light incident upon a cavity containing semiconductor QWs in the configuration of four subbands is shown in Fig. 1. The cavity consists of three layers of materials: two nonmagnetic dielectric slabs $\left(\varepsilon_{1}\right)$ with the same thickness $d_{1}$ and a QW medium $\left(\varepsilon_{2}\right)$ with thickness $d_{2}$; see Fig. 1(a). The asymmetric QW (intracavity medium) structure with the relevant conduction band levels and wave function is shown in Fig. 1(b), which can be grown in the sequence from left to right. $\mathrm{A}$ thick $\mathrm{Al}_{0.4} \mathrm{Ga}_{0.6}$ As barrier is followed by a $\mathrm{Al}_{0.16} \mathrm{Ga}_{0.84}$ As shallow well $(6.8 \mathrm{~nm})$ that is separated from the GaAs deep well $(7.7 \mathrm{~nm})$ on the right by a $\mathrm{Al}_{0.4} \mathrm{Ga}_{0.6}$ As potential barrier $(3.0 \mathrm{~nm})$. Subsequently, a thin $(1.5 \mathrm{~nm})$ $\mathrm{Al}_{0.4} \mathrm{Ga}_{0.6} \mathrm{As}$ barrier separates the deep well from the last thick layer of $\mathrm{Al}_{0.16} \mathrm{Ga}_{0.84} \mathrm{As}$ on the right. The energies of the deep well ground state $|1\rangle$ and the shallow well first excited state $|4\rangle$ are $E_{1}=46.7 \mathrm{meV}$ and $E_{4}=296.3 \mathrm{meV}$, respectively. Two closely spaced delocalized intermediated states $|2\rangle$ and $|3\rangle$, with

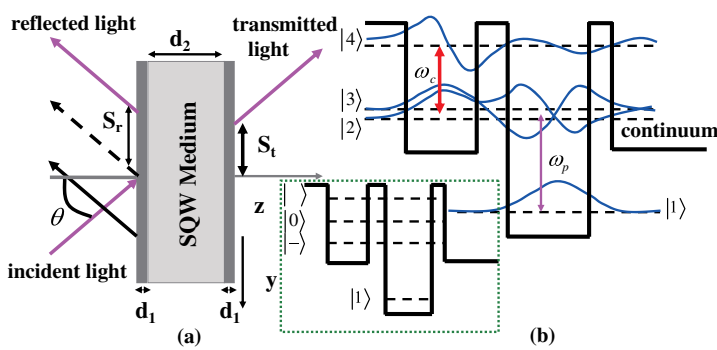

Fig. 1. (a) Schematic of a weak probe light incident upon a cavity containing four-subband QWs, in which the light can be reflected back and transmitted through the cavity with corresponding negative or positive lateral shifts. (b) Schematic band diagram of the asymmetric semiconductor QWs with a deep well and a shallow well. Related subbands and the corresponding wave functions are illustrated by dashed and solid lines. Inset: dressed picture. 
energies $E_{2}=174.8 \mathrm{meV}$ and $E_{3}=183.5 \mathrm{meV}$, are created by mixing the ground state in the shallow well and the first excited state in the deep well via the resonant tunneling. For simplicity, the coherent control beam is assumed to be far away from the resonant modes of the cavity, so that the control beam $\left(\omega_{c}\right)$ could be applied homogeneously onto the transitions $|2\rangle \leftrightarrow|4\rangle$ and $|3\rangle \leftrightarrow|4\rangle$. Because of the selection rule, intersubband absorption occurs only for light polarized perpendicular to the plane of the QW layers [24]. We consider a TE-polarized weak probe light incident on a cavity with an angle $\theta$ to the $z$ axis. The weak probe light $\omega_{p}$ is applied simultaneously to the transitions $|1\rangle \leftrightarrow|2\rangle$ and $|1\rangle \leftrightarrow|3\rangle$.

Under the rotating-wave and dipole approximations, the equations of motion can be written as $(\hbar=1)$

$\dot{A}_{1}=i g \Omega_{p}^{*} A_{2}+i \Omega_{p}^{*} A_{3}$,

$\dot{A}_{2}=i\left(i \gamma_{2}-\Delta_{p}+\Delta\right) A_{2}+i g \Omega_{p} A_{1}+i f \Omega_{c}^{*} A_{4}+\zeta A_{3}$,

$\dot{A}_{3}=i\left(i \gamma_{3}-\Delta_{p}-\Delta\right) A_{3}+i \Omega_{p} A_{1}+i \Omega_{c}^{*} A_{4}+\zeta A_{2}$,

$\dot{A}_{4}=i\left(i \gamma_{4}-\Delta_{p}-\Delta_{c}\right) A_{4}+i f \Omega_{c} A_{2}+i \Omega_{c} A_{3}$,

where $\Delta_{p}=\left(\frac{E_{1}+E_{3}}{2}-E_{1}\right)-\omega_{p}, \Delta_{c}=\left(E_{4}-\frac{E_{1}+E_{3}}{2}\right)-\omega_{c}$ with $\omega_{i}(i=p, c)$ is the angular frequency of the relevant light, whereas $2 \Delta=E_{3}-E_{2}$ represents energy splitting between $|2\rangle$ and $|3\rangle$, determined by the coupling strength of the tunneling. Here, $\Omega_{i}(i=p, c)$ is one-half Rabi frequency for the relevant transitions, i.e., $\Omega_{p}=\mu_{31} E_{p} / 2 \hbar$ and $\Omega_{c}=\mu_{34} E_{c} / 2 \hbar$ with $E_{p, c}$, the slowly varying electric field amplitude of the corresponding light; $g=\mu_{21} / \mu_{31}$ and $f=\mu_{24} / \mu_{34}$ represent the ratio between the relevant transition dipole moments with $\mu_{i, j}(i, j=1-4 ; i \neq j)$ being the dipole moment for the transitions. Moreover, the total decay rates are defined as $\gamma_{i}(i=1-4)=\gamma_{i l}+\gamma_{i d}$, with $\gamma_{i l}$ the population decay rates and $\gamma_{i d}$ the pure dipole dephasing rates. For temperatures up to $10 \mathrm{~K}$ with electric density as low as $10^{24} \mathrm{~m}^{-3}$ [24], the dephasing rates can be estimated to be $\gamma_{2 d}=0.68 \mathrm{meV}, \gamma_{3 d}=$ $0.8 \mathrm{meV}$, and $\gamma_{4 d}=0.5 \mathrm{meV}$, whereas $\gamma_{j l}(j=2,3,4)$ can be calculated by solving the effective mass Schrödinger equations. For the present QW structure, the results turn out to be $\gamma_{2 l}=3.47 \mathrm{meV}, \gamma_{3 l}=4.13 \mathrm{meV}$, and $\gamma_{4 l}=0.8 \mathrm{meV}$. Finally, the term $\zeta=\sqrt{\gamma_{2 l} \cdot \gamma_{3 l}}$ denotes a cross-coupling term between the states $|2\rangle$ and $|3\rangle$ coming from the tunneling to the electronic continuum [17-24]. Further, the term $p=$ $\zeta / \sqrt{\gamma_{2} \cdot \gamma_{3}}=0.83$ represents the strength of Fano-type interference induced by the strong tunneling. The parameter $p$ corresponds to the strength of interference, i.e., there is no interference when $p=0$, while for perfect interference $p=1$.

In an appropriate frame, we can obtain straightforwardly the steady state solutions of Eq. (1) and the slowly varying parts of the polarization in the weak probe light, i.e., $P=$ $\varepsilon_{0} \chi\left(\omega_{p}\right) E=2 N\left(\mu_{21} A_{2} A_{1}^{*}+\mu_{31} A_{3} A_{1}^{*}\right)$, with $N$ being the electron concentration. The expression for the susceptibility $\chi\left(\omega_{p}\right)$ can be written as

$$
\chi\left(\omega_{p}\right)=\frac{i \beta\left[\alpha_{1} \gamma_{4}+(f-g)^{2} \Omega_{c}^{2}\right]}{\alpha_{2} \gamma_{4}+\alpha_{3} \Omega_{c}^{2}},
$$

where $\alpha_{1}=-i \Delta+i g^{2} \Delta+2 g \zeta+\gamma_{2}+g^{2} \gamma_{3}, \alpha_{2}=\Delta^{2}-\zeta^{2}-$ $i \Delta \gamma_{3}+\gamma_{2}\left(i \Delta+\gamma_{3}\right), \alpha_{3}=-i \Delta+i f^{2} \Delta+2 f \zeta+\gamma_{2}+f^{2} \gamma_{3}$, and $\beta=N \mu_{31}^{2} / \varepsilon_{0} \hbar$. The susceptibility $\chi\left(\omega_{p}\right)$ and permittivity $\varepsilon_{2}$ of the QW medium can therefore be related to each other via the relation $\varepsilon_{2}=1+\chi\left(\omega_{p}\right)$.

For the incident probe beam with a large width (i.e., the beam with a narrow angular spectrum [25]), the GH shifts of both the reflected and transmitted beams can be expressed using a stationary phase theory $[\underline{9}, \underline{26}]$

$$
S_{r, t}=\xi\left[\operatorname{Re}(r, t) \frac{d \operatorname{Im}(r, t)}{d \theta}-\operatorname{Im}(r, t) \frac{d \operatorname{Re}(r, t)}{d \theta}\right],
$$

where $\xi=-\lambda / 2 \pi|r, t|^{2}$ and $r$ and $t$ are the reflection and transmission coefficients, respectively. To find the GH shift, reflection and transmission coefficients should be calculated first. To calculate the reflection and transmission coefficients, we apply the standard characteristic matrix approach $[27,28]$ for the weak probe beam through the cavity. The transfer matrix of the $j$ th layer can be expressed as

$$
M_{j}\left(k_{y}, \omega_{p}, d_{j}\right)=\left(\begin{array}{cc}
\cos \left(k_{z}^{j} d_{j}\right) & i \sin \left(k_{z}^{j} d_{j}\right) / q_{j} \\
i q_{j} \sin \left(k_{z}^{j} d_{j}\right) & \cos \left(k_{z}^{j} d_{j}\right),
\end{array}\right),
$$

where $k_{z}^{j}=\sqrt{\varepsilon_{j} k^{2}-k_{y}^{2}}$ is the $z$-component of the wave number in the $j$ th layer, $q_{j}=k_{z}^{j} / k, k=\omega_{p} / c, d_{j}$ is the thickness of the $j$ th layer, $k_{y}$ is the $y$-component of the wave number in vacuum, and $c$ is the light speed in vacuum. The total transfer matrix for three layers can be written as $Q\left(k_{y}, \omega_{p}\right)=$ $M_{1}\left(k_{y}, \omega_{p}, d_{1}\right) M_{2}\left(k_{y}, \omega_{p}, d_{2}\right) M_{3}\left(k_{y}, \omega_{p}, d_{1}\right)$. Subsequently, the reflection and transmission coefficients are, respectively, obtained by using the total transfer matrix:

$$
\begin{aligned}
& r\left(k_{y}, \omega_{p}\right)=\frac{q_{0}\left(Q_{22}-Q_{11}\right)-\left(q_{0}^{2} Q_{12}-Q_{21}\right)}{q_{0}\left(Q_{22}+Q_{11}\right)-\left(q_{0}^{2} Q_{12}+Q_{21}\right)}, \\
& t\left(k_{y}, \omega_{p}\right)=\frac{2 q_{0}}{q_{0}\left(Q_{22}+Q_{11}\right)-\left(q_{0}^{2} Q_{12}+Q_{21}\right)},
\end{aligned}
$$

where $q_{0}=k_{z} / k$ with $k_{z}$ the $z$-component of the wave number in vacuum, and $Q_{i j}$ is the element of the matrix $Q\left(k_{y}, \omega_{p}\right)$. Based on Eq. (3), we can analyze theoretically the influence of tunneling-induced interference on the GH shifts in the reflected and transmitted probe beams. The parameters $\Delta_{p}=$ $\Delta_{c}=0, \beta=0.0184 \mathrm{meV}, d_{1}=2 \mu \mathrm{m}, d_{2}=50 \mu \mathrm{m}, \varepsilon_{1}=$ 2.22 , and $\omega_{p}=132.45 \mathrm{meV}$ (corresponding the wavelength $\lambda=9.3 \mu \mathrm{m})$ remain fixed for the system. Because of the resonant tunneling between the ground level of the shallow well and the first excited level of the deep well, the wave functions of subbands $|2\rangle$ and $|3\rangle$ are symmetric and antisymmetric combinations of those associated with the isolated deep and shallow wells, and we can take $g=-f=-1$ in Eq. (1).

When the control beam is off $\left(\Omega_{c}=0\right)$, the four-state system may be decoupled to a three-state system consists of $|1\rangle$, $|2\rangle$, and $|3\rangle$. Accordingly, the susceptibility $\chi\left(\omega_{p}\right)$ in Eq. (2) can be simplified to $\chi\left(\omega_{p}\right)=i \beta \alpha_{1} / \alpha_{2}$. In this case, the tunneling-induced interference $\zeta$ plays a key role in modifying the susceptibility and permittivity for a fixed energy splitting $\Delta$. For small dephasing rates, i.e., $\gamma_{2 d}=0.68 \mathrm{meV}$ and $\gamma_{3 d}=0.8 \mathrm{meV}$, the value $p=0.83$ is close to the ideal value $p=1$ and corresponds to a large tunneling efficiency, leading to a perfect interference. As observed in Fig. 2(a), the maximum $\mathrm{GH}$ shifts in the reflected and transmitted beams are located in $\theta=0.74$ and $\theta=1.3 \mathrm{rad}$, respectively. Accordingly, the achievable maximum values of negative and positive $\mathrm{GH}$ shifts 

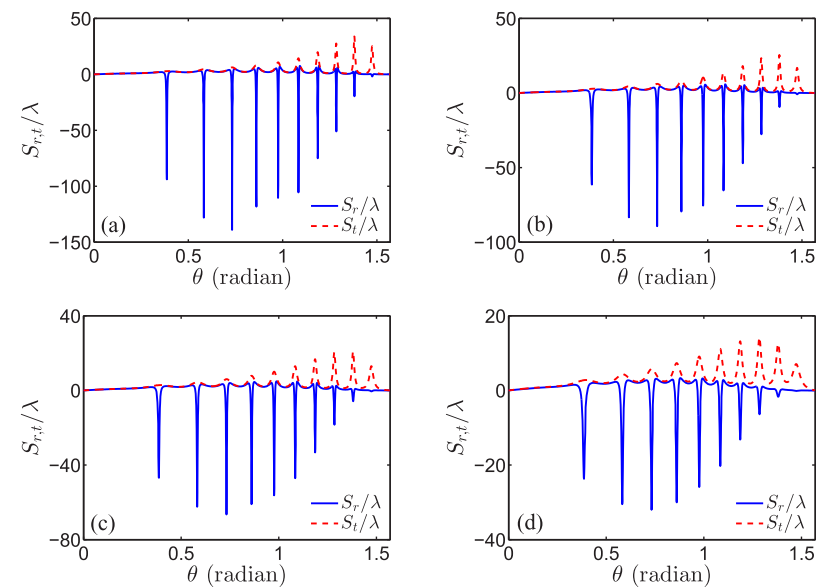

Fig. 2. GH shift $S_{r, t} / \lambda$ of reflected and transmitted probe beams versus $\theta$ for (a) $\Omega_{c}=0$; (b) $\Omega_{c}=0, \gamma_{2 d}=1.36 \mathrm{meV}$, and $\gamma_{3 d}=1.6 \mathrm{meV}$; (c) $\Omega_{c}=1 \mathrm{meV}$; and (d) $\Omega_{c}=6 \mathrm{meV}$. Other parameters used are given in the text.

might be $140 \lambda \sim 1.31 \mathrm{~mm}$ and $35 \lambda \sim 0.36 \mathrm{~mm}$. The value of interference strength $p$ can be reduced by a temperature increase (up to $30 \mathrm{~K}$ [24]) which generally leads to larger dephasing rates (i.e., $\gamma_{2 d}=1.36 \mathrm{meV}$ and $\gamma_{3 d}=1.6 \mathrm{meV}$ ). In this case, the maximum values of GH shifts in the reflected and transmitted beams are reduced significantly; see Fig. 2(b). The effects of Fano-type interference induced by the tunneling on the GH shifts can readily be explained physically. When the dephasing rates $\gamma_{i d}$ become large, the effects of tunnelinginduced interference start to become less pronounced, which results into a resonant probe being more and more absorbed and the GH shifts being reduced. On the other hand, when the control beam is on $\left(\Omega_{c} \neq 0\right)$, the coupling transitions $|2\rangle \leftrightarrow|4\rangle$ and $|3\rangle \leftrightarrow|4\rangle$ lead to enhanced probe absorption of the intercavity medium $(\mathrm{QW})$ via quenching of the tunnelinginduced interference. In the presence of the control beam, it can be found from Figs. 2(c) and 2(d) that the GH shifts in the reflected and transmitted beams become obviously smaller than those shown in Figs. 2(a) and 2(b). In addition, Figs. 2(c) and $2(\mathrm{~d})$ show that the maximum values of GH shifts depend on the external control beam strength.

The effects of the control beam on the GH shifts can be explained in terms of dressed states picture. Taking $|a\rangle(|b\rangle)$ as the first excited (ground) state of the deep (shallow) well, when they are isolated by an infinitely thick barrier, the states split into a doublet $|2\rangle=(|a\rangle+|b\rangle) / \sqrt{2}$ and $|3\rangle=(-|a\rangle+$ $|b\rangle) / \sqrt{2}$ in the case of resonant tunneling. Since $\langle a|\mu| 1\rangle$ is the only nonvanishing probe dipole matrix element, it follows that subbands $|2\rangle$ and $|3\rangle$ are both coupled by the probe to the ground with $\mu_{21} \equiv\langle 2|\mu| 1\rangle=-\mu_{31}=\mu_{a 1} / \sqrt{2}$. Similarly, for the control beam, we have $\mu_{42}=\mu_{43}=\mu_{4 b} / \sqrt{2}$. In the case of $\Delta_{c}=0$, the control beam drives the transitions $|2\rangle \leftrightarrow|4\rangle$ and $|3\rangle \leftrightarrow|4\rangle$, and generates three dress states $|0\rangle$ and $| \pm\rangle$ as shown in the inset of Fig. 1 . The dress state $|0\rangle$ is given by $|0\rangle=$ $\frac{\Omega_{c}}{\sqrt{2 \Omega_{c}^{2}+\Delta^{2}}}(|2\rangle-|3\rangle)-\frac{\Delta}{\sqrt{2 \Omega_{c}^{2}+\Delta^{2}}}|4\rangle$, with eigenfrequency $\omega_{0}=$ $\left(E_{2}+E_{3}\right) / 2$. The state $|0\rangle$ may couple to the ground state $|1\rangle$ causing the absorption of a resonant probe. This new dipole allowed transition $|0\rangle \leftrightarrow|1\rangle$ induced by the control beam has a dipole moment $\mu_{01}=\mu_{a 1} \Omega_{c} / \sqrt{2 \Omega_{c}^{2}+\Delta^{2}}$, which depends on $\Delta$ and $\Omega_{c}$. As a consequence, the probe absorption increases as $\Omega_{c}$ increases, which leads to the suppression of the GH shifts in the incident probe beam; see Figs. 2(c) and 2(d). This control beam opens a new interference pathway and destroys the tunneling-induced interference. As a matter of fact, this is a consequence from the competition between the destructive interference induced by the coherent control beam and the Fanotype interference induced by the resonant tunneling.

For a direct insight on the above mentioned competition behavior between two-type interferences that modify the GH shifts, the contour maps of the GH shifts in the transmitted and reflected beams versus $\Omega_{c}$ and $\theta$ are shown in Figs. 3(a) and $3(\mathrm{~b})$, respectively. It can be seen from Fig. 3 that one maximum value of the GH shifts occurs, whether reflected (negative) or transmitted (positive) beams, which agrees with the results shown in Fig. 2. For a fixed incident angle $\theta$, Fig. 3 also shows that the GH shifts in the transmitted and reflected beams decrease monotonically as $\Omega_{c}$ increases. Furthermore, after a critical value of $\Omega_{c}(\approx \Delta / \sqrt{2} \approx 3 \mathrm{meV})$, the negative and positive $\mathrm{GH}$ shifts remain unchanged as $\Omega_{c}$ increases continuously. By increasing the intensity of the control beam $\left(\Omega_{c}>3 \mathrm{meV}\right)$, the competition of the two-type interferences makes the probe absorption on the transitions $|1\rangle \leftrightarrow|2\rangle$ and $|1\rangle \leftrightarrow|2\rangle$ reach the saturation.

Based on the analysis of dressed states, one can find that the coupling strengths of resonant tunneling $\Delta$ plays an important role in modifying the tunneling-induced interference, which can lead to an effective control of the GH shifts. We show the GH shifts $S_{r, t}$ in the reflected and transmitted beams versus incident angle $\theta$ for different values of $\Delta$ when $\Omega_{c}=0$, and demonstrate that the GH shifts have a sensitive dependence on $\Delta$; see Fig. $\underline{4}$. The achievable maximum values of $\mathrm{GH}$ shifts in the reflected and transmitted beams can reach $280 \lambda \sim$ $2.62 \mathrm{~mm}$ and $60 \lambda \sim 0.56 \mathrm{~mm}$, respectively. Interestingly, the splitting induced by resonant tunneling can be adjusted via
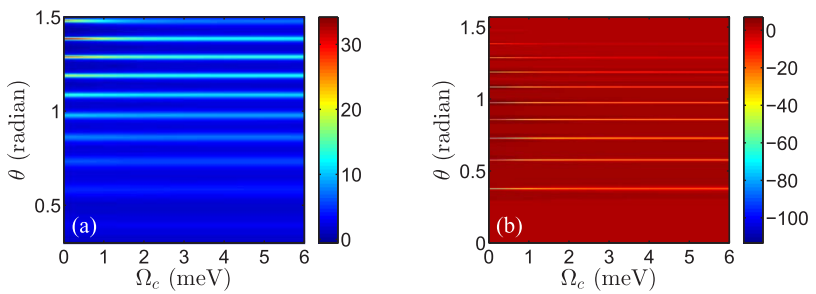

Fig. 3. Contour maps of the GH shift in the transmitted and reflected probe beams versus $\Omega_{c}$ and $\theta$ : (a) $S_{t} / \lambda$ and (b) $S_{r} / \lambda$. Other parameters are the same as in Fig. $\underline{2(a)}$.
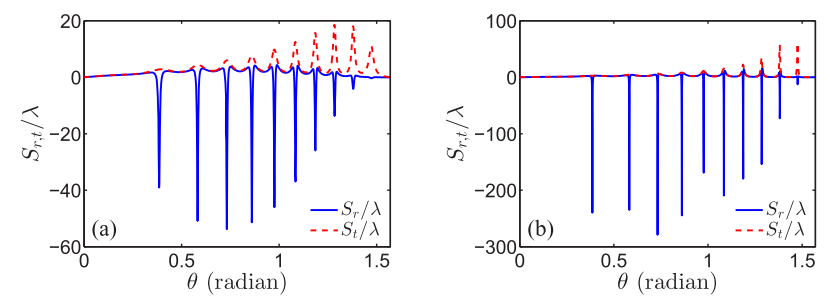

Fig. 4. GH shift $S_{r, t} / \lambda$ of reflected and transmitted probe beams versus $\theta$ for (a) $\Delta=2 \mathrm{meV}$ and (b) $\Delta=8 \mathrm{meV}$. Other parameters are the same as in Fig. 2(a). 

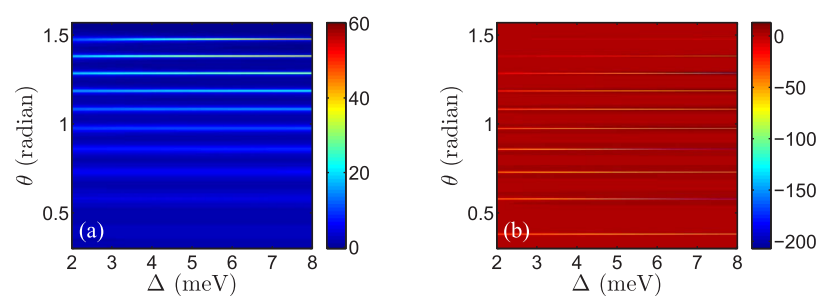

Fig. 5. Contour maps of the GH shift in the transmitted and reflected probe beams versus $\Delta$ and $\theta$ : (a) $S_{t} / \lambda$ and (b) $S_{r} / \lambda$. Other parameters are the same as in Fig. 2(a).

external bias, which can be engineered to give a desired coupling strength of resonant tunneling by utilizing so-called structure coherent control [24]. As a result, we may provide a novel method to manipulate the GH shifts in a convenient way. To see a more clear picture of enhanced $\mathrm{GH}$ shifts in the reflected and transmitted light by varying $\Delta$, the contour maps of the GH shifts in the transmitted and reflected beams versus $\Delta$ and $\theta$ are shown in Figs. 5(a) and 5(b), respectively. For a fixed incident angle $\theta$, Fig. 5 also shows that the GH shifts in the transmitted and reflected beams increase linearly proportional to $\Delta$.

In conclusion, we have presented a scheme to control the $\mathrm{GH}$ shifts in the reflected and transmitted beams when a light is incident on a cavity containing a QW structure, by means of modulating the optical properties of the intercavity medium. With Schrödinger formalism and transfer matrix, we have derived explicitly the corresponding expressions of $\mathrm{GH}$ shifts in the reflected and transmitted light and showed that the GH shifts in the reflected and transmitted lights can be controlled via an external control beam and the tunable coupling strength of resonant tunneling. Our results illustrate the potential to utilize tunneling-induced interference for optimizing the $\mathrm{GH}$ shifts, as well as a guidance in the design of optical devices in sensors and information processing.

Ministry of Science and Technology, Taiwan (MOST); National Natural Science Foundation of China (NSFC) (11374050, 61372102).

\section{REFERENCES}

1. F. Goos and H. Hänchen, Ann. Phys. 436, 333 (1947).

2. T. Hashimoto and T. Yoshino, Opt. Lett. 14, 913 (1989).

3. X. Hu, Y. Huang, W. Zhang, D. K. Qing, and J. Peng, Opt. Lett. 30, 899 (2005).

4. G. Y. Oh, D. G. Kim, and Y. W. Choi, Opt. Express 17, 20714 (2009).

5. L. G. Wang, M. Ikram, and M. S. Zubairy, Phys. Rev. A 77, 023811 (2008).

6. Ziauddin, S. Qamar, and M. S. Zubairy, Phys. Rev. A 81, 023821 (2010).

7. Ziauddin and S. Qamar, Phys. Rev. A 84, 053844 (2011).

8. Ziauddin, Y. L. Chuang, and R.-K. Lee, Phys. Rev. A 91, 013803 (2015).

9. L. G. Wang, H. Chen, and S. Y. Zhu, Opt. Lett. 30, 2936 (2005).

10. Y. Wu and X. Yang, Phys. Rev. A 71, 053806 (2005).

11. M. Fleischhauer, A. Imamoglu, and J. P. Marangos, Rev. Mod. Phys. 77, 633 (2005).

12. G. S. Agarwal, Phys. Rev. Lett. 67, 980 (1991).

13. S. E. Harris, Phys. Rev. Lett. 62, 1033 (1989).

14. Y. Wu, L. Wen, and Y. Zhu, Opt. Lett. 28, 631 (2003).

15. L. J. Wang, A. Kuzmich, and A. Dogariu, Nature 406, 277 (2000).

16. L. V. Hau, S. E. Harris, Z. Dutton, and C. H. Behroozi, Nature 397, 594 (1999).

17. J. Faist, F. Capasso, C. Sirtori, K. W. West, and L. N. Pfeiffer, Nature 390, 589 (1997).

18. H. Schmidt, K. L. Campman, A. C. Gossard, and A. Imamoglu, Appl. Phys. Lett. 70, 3455 (1997).

19. G. B. Serapiglia, E. Paspalakis, C. Sirtori, K. L. Vodopyanov, and C. C. Phillips, Phys. Rev. Lett. 84, 1019 (2000).

20. J. H. Wu, J. Y. Gao, J. H. Xu, L. Silvestri, M. Artoni, G. C. LaRocca, and F. Bassani, Phys. Rev. Lett. 95, 057401 (2005).

21. H. Sun, Y. Niu, R. Li, S. Jin, and S. Gong, Opt. Lett. 32, 2475 (2007).

22. W. X. Yang, J. M. Hou, and R.-K. Lee, Phys. Rev. A 77, 033838 (2008).

23. W. X. Yang, J. M. Hou, Y. Y. Lin, and R.-K. Lee, Phys. Rev. A 79, 033825 (2009).

24. H. C. Liu and F. Capasso, Intersubband Transitions in Quantum Wells: Physics and Device Applications (Academic, 2000), pp. 5-18.

25. K. Artmann, Ann. Phys. 437, 87 (1948).

26. C. F. Li, Phys. Rev. Lett. 91, 133903 (2003).

27. M. Born and E. Wolf, Principles of Optics, 7th ed. (Cambridge University, 1999).

28. N. H. Liu, S. Y. Zhu, H. Chen, and X. Wu, Phys. Rev. E 65, 046607 (2002). 